Gastroenterología, Hospital Clínico de la Universidad de Chile. ${ }^{2}$ Unidad Gastroenterología, Clínica Santa María. aecaria Gastroenterología, Hospital Clínico de la Universidad de Chile.

Recibido el 28 de julio de 2011, aceptado el 27 de octubre de 2011.

Correspondencia a: Dr. Jaime Poniachik Teller, Centro de Gastroenterología, Hospital Clínico José Joaquín Aguirre, Universidad de Chile. Santos Dumont No 999, Santiago. Teléfono: 6788350. Fax: 6788349 .

\section{Ligadura endoscópica, una nueva opción terapéutica para la ectasia vascular antral: presentación de dos casos clínicos}

\author{
JAIME PONIACHIK T. ${ }^{1,2}$, ZOLTÁN BERGER F. ${ }^{1}$, ARAXI MANUGUIÁN G. ${ }^{\text {a }}$
}

Gastric antral vascular ectasia is an uncommon cause of chronic anemia, occasionally associated with cirrhosis. The most accepted therapy is argon plasma coagulation (APC), however there are refractory cases. We report two females with cirrhosis, aged 60 and 72 years, in whom management with APC was insufficient and in whom the need for hospital admissions and transfusions were reduced using the technique of endoscopic band ligation.

(Rev Med Chile 2012; 140: 364-367).

Key words: Argon plasma coagulation; Gastric antral vascular ectasia; Gastrointestinal hemorrhage.

L a ectasia vascular antral (EVA) es una lesión vascular poco común, responsable de alrededor de $4 \%$ de las hemorragias digestivas altas (HDA) no variceales ${ }^{1-3}$.

Descrita por primera vez en 1953 por Rider como "un tipo de gastritis erosiva con marcada ectasia veno-capilar"4-5. En 1984, Jabbari la llama watermelon stomach debido a los tractos longitudinales antrales que convergen hacia el píloro y que contienen ectasias vasculares y que recuerdan a las líneas de una sandía ${ }^{4-6}$. La mayoría son idiopáticas, otras se han asociado a cirrosis, esclerosis sistémica, insuficiencia renal e hipotiroidismo y otras enfermedades del tejido conectivo ${ }^{7}$.

Predomina en mayores de 70 años, presentándose como anemia crónica, hasta 4\% de ellos presentan anemia de difícil manejo, requiriendo transfusiones ${ }^{3-8}$.

La patogenia es desconocida, pero podría obedecer a cambios crónicos en la vasculatura de la mucosa y submucosa en respuesta a traumas reiterados y gatillados por las contracciones antrales probablemente secundarias a denervación del sistema autónomo (disfunción motora antropilórica $)^{8}$.
Además se ha encontrado aumento de células neuroendocrinas productoras de polipéptido intestinal vasoactivo y 5 - hidroxitriptamina, que serían al menos en parte responsables de la ectasia vascular $^{9,10}$.

El diagnóstico es endoscópico y se puede confirmar con histología.

La histología característica incluye ectasia vascular intramucosa, trombos de fibrina, fibrohialinosis e hipertrofia fibromuscular de la lámina propia, hallazgos muy similares a los encontrados en la intususcepción, vólvulo y trauma, lo que apoya la teoría de la disfunción motora antropilórica $^{1-3,4-7}$.

La terapia incluye diferentes técnicas endoscópicas como coagulación endoscópica con diferentes elementos, en particular con argón plasma (APC), que luego de sesiones reiteradas reduce el sangrado ${ }^{11}$. En casos refractarios se plantea la antrectomía $^{12}$.

Presentamos dos casos de fracaso en el manejo con APC (según técnica estandarizada con equipo EMED-ES-350 con un flujo de 1,5 y potencia de $60 \mathrm{~W}$ ) en los que se aplicaron ligaduras al antro. 


\section{Casos clínicos}

\section{Caso 1}

Mujer de 60 años con cirrosis hepática por alcohol Child-Pugh B diagnosticada el año 2004 con várices esofágicas ligadas y erradicadas.

Consultó en nuestro centro en 2009 por desorientación, disnea y astenia, demostrándose anemia severa con hemoglobina de 4,2 g/dL. Una primera endoscopia digestiva alta (EDA) mostró EVA tipo watermelon stomach manejándose con APC y transfusiones de plaquetas, plasma y glóbulos rojos, en unidad de cuidados intermedios.

Dos meses después reinició la disnea, por lo que se hizo nueva EDA que confirmó la EVA, realizándose una nueva sesión de APC.

A los 3, 5 y 6 meses presentó otros tres episodios de hematemesis, requiriendo en todos hospitalización, transfusiones y EDA, encontrando siempre la EVA sangrando por lo que se hicieron 3 sesiones más de APC.

De la última hospitalización se dio el alta programando un control endoscópico ambulatorio en el que se encontró un estómago con sangre fresca y EVA, realizando ligadura endoscópica con 8 bandas.

Un mes después una nueva EDA mostró una franca reducción del aspecto de watermelon stomach, realizando nuevas ligaduras al antro.

\section{Caso 2}

Mujer de 72 años con cirrosis por esteatohepatitis no alcohólica Child-Pugh B diagnosticada el año 2004.
Consultó en el año 2008 en Clínica Santa María cuando se hospitalizó por ascitis; se diagnosticó un hepatocarcinoma de $3 \mathrm{~cm}$, mediante un scanner de abdomen, se realizó terapia con radiofrecuencia. Luego ingresó en varias oportunidades por encefalopatía grado II y anemia con hematocrito de $24 \%$. Una primera endoscopia digestiva alta (EDA) mostró EVA tipo watermelon stomach manejándose con APC, luego se hizo nueva EDA que confirmó la EVA, realizándose una ligadura antral por mala respuesta endoscópica. Luego de varios meses se repitió la EDA mostrando mejoría significativa de la EVA, realizándose una segunda sesión de ligaduras. En endoscopias posteriores no se evidencia EVA. La paciente falleció en abril de 2011 por un cuadro séptico de origen pulmonar (Figuras 1 y 2).

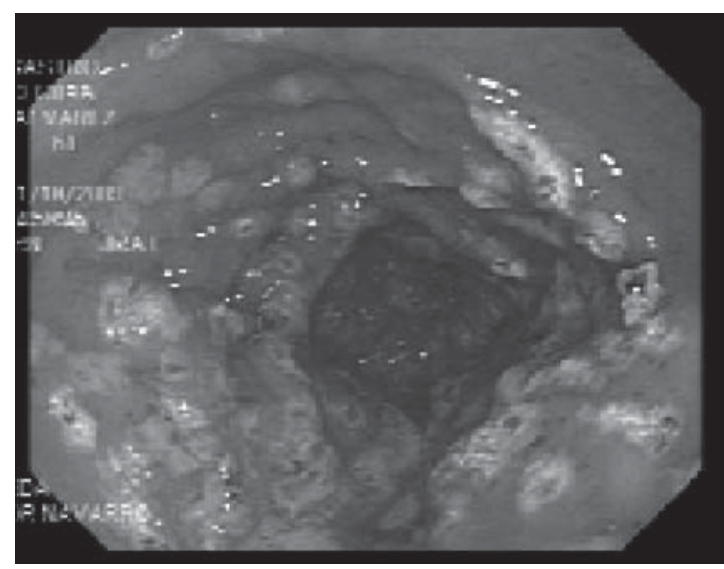

Figura 1. Aspecto endoscópico posterior a terapia con APC, Caso clínico 1.
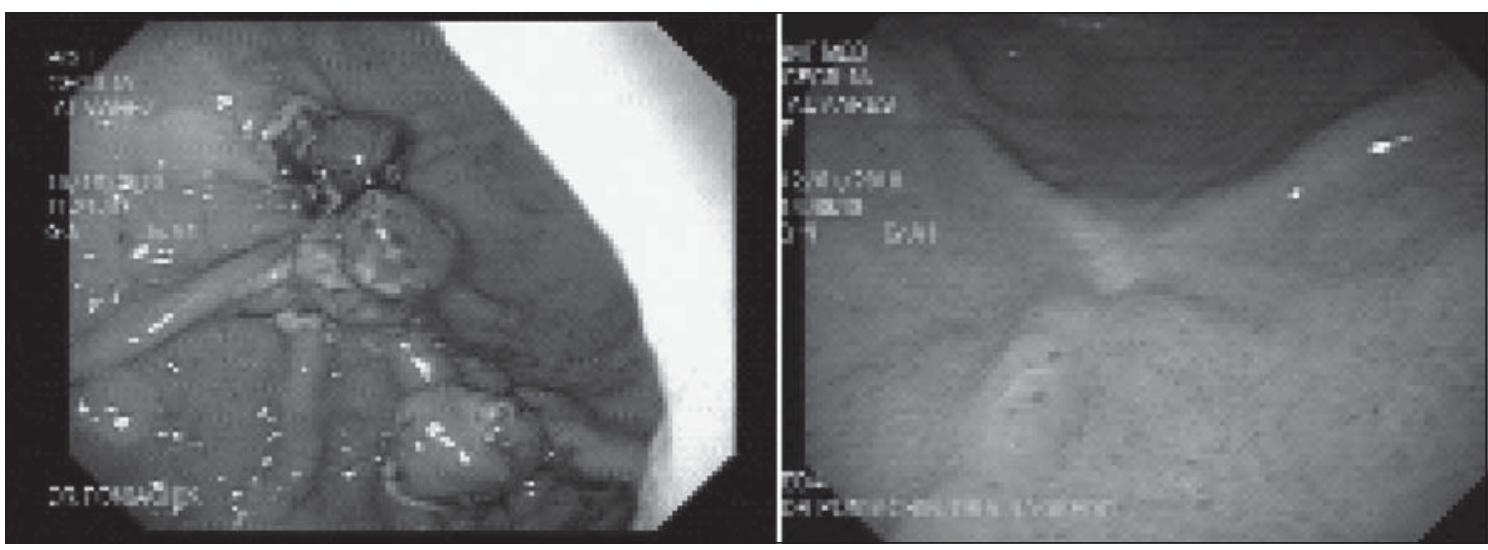

Figura 2. Ligaduras endoscópicas al antro y sus cicatrices en el control endoscópico un mes después, Caso clínico 1. 


\section{Discusión}

El diagnóstico de EVA es infrecuente, pero representa un desafío terapéutico pues implica en la mayoría de los casos la persistencia de anemia que deteriora la calidad de vida del paciente y en otros menos, la necesidad de transfusiones, incluso poniendo en riesgo la vida del paciente debido a lo severo de las anemias.

Se ha descrito con anterioridad una latencia diagnóstica de hasta 4 años, pues cuando es inicial existe la posibilidad de confundir la imagen endoscópica con otro tipo de gastropatías, requiriendo de una biopsia para la confirmación diagnóstica, lo que no siempre se realiza. En nuestros casos la biopsia se obvió debido al aspecto muy sugerente de EVA.

Nuestras pacientes se presentaron en el rango de edad descrito y además cursaban con cirrosis hepática.

Si pensamos en la hipótesis de la denervación vagal con hipertonía simpática, la EVA de la cirrosis podría estar relacionada con la hiperactividad simpática que también caracteriza a la disfunción cardiaca y renal de estos pacientes ${ }^{1}$.

La disfunción autonómica podría ser un factor común entre todas las patologías relacionadas con la EVA, lo que representa un tópico interesante a investigar ${ }^{9,10}$.

Aproximadamente 4 años después de diagnosticar la cirrosis, nuestra primera paciente presentó anemia severa y muy sintomática, encontrando en ese momento la EVA, que se encontraba asociada a várices pequeñas. En su evolución posterior la anemia y los episodios de hemorragia digestiva alta severa fueron recurrentes, manejándose siempre con transfusiones y sesiones de APC, reanudando los síntomas y la anemia, por lo que se decidió tratar con otra terapia, realizando ligaduras del antro, lo que resultó técnicamente simple y con resultados inmediatos satisfactorios.

Respecto de la terapia de la EVA, se han intentado diferentes técnicas que se podrían clasificar en tres grupos: farmacológicas, endoscópicas y quirúrgicas.

Dentro de las farmacológicas se han usado corticoides, combinaciones de estrógeno-progesterona, talidomida, interferón alfa, calcitonina y ciproheptadina, pero todos estos han sido reportes de casos aislados, sin lograr ser reproducidos en series más grandes ${ }^{1-12}$.

El uso de beta bloqueadores o TIPS para reducir la hipertensión portal no ha mostrado beneficio $^{2-7}$.

Para casos severos y cuando falla la terapia endoscópica, siempre queda el recurso terapéutico de la antrectomía, aunque existe consenso en que no debe ser la terapia de elección pues tiene alta morbimortalidad ${ }^{12}$.

Las terapias endoscópicas, iniciadas en 1980, son las más recomendadas pues han demostrado eficacia y seguridad, incluyendo diferentes técnicas (electrocoagulación, APC, escleroterapia, ligadura endoscópica), incluso se publicó un caso de resección mucosa de la EVA, con éxito y mejoría de la anemia ${ }^{13}$.

Los trabajos realizados con APC muestran una reducción de la necesidad de transfusión en hasta $77 \%$ durante un seguimiento de 16 meses, siendo endoscópicamente satisfactoria hasta en 80 a 90\% de los casos $^{4-14}$.

Sin embargo, lo habitual es que requieran varias sesiones (en la literatura se reporta un promedio de 6 sesiones para lograr curar la mucosa) y pueden fallar cuando las lesiones son muy difusas ${ }^{15}$.

Parece ser el caso de nuestras pacientes, en quienes a pesar de varias sesiones de APC, con la técnica habitual y características técnicas ya descritas, reiteraban las hospitalizaciones por anemia sintomática e incluso hemorragia digestiva alta severa, por lo que se intentó el manejo con ligadura endoscópica.

Debemos indicar que la técnica y características de procedimiento terapéutico con APC, fue el mismo en todas las sesiones, sin efectuar cambios en el flujo del gas ni potencia, por lo que nuestro trabajo no permite descartar un eventual cambio en el resultado de haber usado variables técnicas diferentes.

La ligadura endoscópica es una técnica universalmente aceptada para el manejo de las várices esofágicas pues ha demostrado la obliteración de los plexos submucosos. También se han usado para tratar otras lesiones como hemorroides y lesiones de Dieulafoy ${ }^{16,17}$.

Cabe suponer un éxito similar en la EVA, pues las alteraciones histológicas y ectasia vascular, se encuentran también en la mucosa y submucosa.

En esto se han basado otros autores que ya han reportado casos de terapia exitosa con esta técnica ${ }^{14-18,19}$.

Wells et al publicaron una serie de 22 pacientes con EVA, comparables en cuanto a datos demográ- 
Ligadura endoscópica en ectasia vascular antral - J. Poniachik et al

ficos, presentación clínica, requerimientos transfusionales y asociación con hipertensión portal. Trece de ellos fueron tratados con APC, los otros 9 fueron sometidos a ligadura endoscópica. Los resultados indicaron que la terapia con ligadura fue mejor, estadísticamente, para detener el sangrado activo, en aumentar la hemoglobina, para reducir el número de transfusiones requeridas y en la reducción del número de hospitalizaciones.

En cuanto a las complicaciones la terapia con APC, si bien es bastante segura, cuenta con reportes de perforaciones y obstrucción al tracto de salida gástrico ${ }^{15}$. No existen reportes de complicaciones para la ligadura endoscópica de la EVA.

La ligadura endoscópica de la EVA lograría una resección de la mucosa y submucosa antral que se encuentra visiblemente afectada ${ }^{13}$.

En el estudio previo, con una sesión con aplicación de 12 ligaduras se logró obtener una mucosa aparentemente sana con mínimas lesiones residuales.

Existen otros dos trabajos con casuísticas mucho menores pero con resultados similares a favor de la ligadura endoscópica ${ }^{18,19}$.

Nuestras pacientes fueron tratadas con ligaduras endoscópicas aplicadas desde la región antropilórica hacia proximal.

En conclusión, mostramos el caso de dos pacientes cirróticas y portadoras de EVA, de difícil manejo, tratadas en varias oportunidades con APC con malos resultados, que logran controlar las complicaciones luego de aplicar ligaduras endoscópicas planteando idealmente la necesidad de realizar estudios prospectivos, controlados y con un número de pacientes que permitan extrapolar los resultados a la práctica clínica habitual.

\section{Referencias}

1. Novitsky W, Kercher W, Czerniach R, Litwin E. Watermelon stomach: pathophysiology, diagnosis, and management. J Gastrointest Surg 2003; 7 (5): 652-61.

2. Dulai S, Jensen M, Kovacs O, Gralnek M, Jutabha R. Endoscopic treatment outcomes in watermelon stomach patients with and without portal hypertension. Endoscopy 2004; 36 (1): 68-72.

3. Nguyen Connie LE, Hanh Nguyen. Gastric Antral Vascular Ectasia (Watermelon Stomach) - An Enigmatic and OftenOverlooked Cause of Gastrointestinal Bleeding in the Elderly. The Permanente Journal Fall 2009; 13 (4): 46-9.

4. Poniachik J, Quera R, Sáenz R, Alfaro J, Smok G. Coa- gulación endoscópica con Plasma de Argón, una opción terapéutica en la ectasia vascular antral. Rev Med Chile 2001; 129 (5): 547-51.

5. Rider A, Klotz P, Kirsner B. Gastritis with veno-capillary ectasia as a source of massive gastric hemorrhage. Gastroenterology 1953; 24: 118-23.

6. Jabbari M, Cherry R, Lough O, Daly S, Kinnear G, Goresdy A. Gastric antral vascular ectasia: the watermelon stomach. Gastroenterology 1984; 87: 1165-70.

7. Spahr L, Villeneuve P, Dufresne P, Tassé D, Bui B, Willems B, et al. Gastric antral vascular ectasia in cirrhotic patients: absence of relation with portal hypertension. Gut 1999; 44: 739-42.

8. Charneau J, Petit R, Calès P, Dauver A, Boyer J. Antral motility in patients with cirrhosis with or without gastric antral vascular ectasias. Gut 1995; 37 (4): 488-92.

9. Vonend O, Rump C, Ritz E. Sympathetic overactivity the Cinderella of cardiovascular risk factors in dialysis patients. Semin Dial 2008; 21(4): 326-30.

10. Lowes R, Rode J. Neuroendocrine cell proliferations in gastric antral vascular ectasia. Gastroenterology 1989; 97: 207-12.

11. Kwan V, Bourke MJ, Williams SJ, Gillespie PE, Murray MA, Kaffes AJ, et al. Argonplasma coagulation in the management of symptomatic gastrointestinal vascular lesions: experience in 100 consecutive patients with longterm follow-up. Am J Gastroenterol 2006; 101: 58-63.

12. Sherman V, Klassen R, Feldman S, Jabbari M, Marcus V, Fried M. Laparoscopic antrectomy: a novel approachto treating watermelon stomach. J Am Coll Surg 2003; 197 (5): 864-7.

13. Kastsinelos P, Chatzimavroudis G, Katsinelos T, Panagiotopolou K, Kotakidou R, Tsolkas G, et al. Endoscopic mucosal resection for recurrent gastric antral vascular ectasia. Vasa 2008; 37 (3): 289-92.

14. Wells C, Harrison E, Gurudu S, Crowell M, Byrne T, DePetris G, et al. Treatment of gastric antral vascular ectasia (watermelon stomach) with endoscopic band ligation. Gastrointestinal Endoscopy 2008; 68 (2): 231-6.

15. Pavey DA, Craig PI. Endoscopic therapy for upper-GI vascular ectasias. Gastrointest Endosc 2004; 59: 233-8.

16. Valera M, Pino Q, Poniachik J, Gil LC, O’Brien M, Sáenz, $\mathrm{R}$, et al. Endoscopic Band Ligation of Bleeding Dieulafoy Lesions: The Best Therapeutic Strategy. Endoscopy 2006; 38: 193-4.

17. McGrath K, Mergener K, Branch S. Endoscopic band ligation of Dieulafoy's lesion: report of two cases and review of the literature. Am J Gastroenterol 1999; 94 (4): 1087-90.

18. Kumar R, Mohindra S, Pruthi. Endoscopic band ligation: a novel therapy for bleeding gastric antral vascular ectasia. Endoscopy 2007; 39 (S): 56-7.

19. Sinha SK, Udawat HP, Varma S, Lal A, Rana SS, Bashin DK. Watermelon stomach treated with endoscopic band ligation. Gastrointest Endosc 2006; 64: 1028-3. 